\title{
High-Order Absorbing Boundary Condition, Domain Decomposition Method and Stratified Dispersive Wave Model
}

\author{
M. Jacques ${ }^{1}$, O. Wilk ${ }^{1}$ \\ Department of Mathematics \\ Modélisation Mathématique et Numérique \\ Conservatoire National des Arts et Métiers \\ 292, rue Saint Martin, Paris, 75003, France
}

\begin{abstract}
The high-order Absorbing Boundary Condition proposed by Hagstrom and Warburton was applied to various models as the wave equation, dispersive, convected with stratified materials. We apply it here to a Stratified Dispersive Wave Model not just to limit the real unbounded domain but to use a non-overlapping domain decomposition method with the classical Schwarz Waveform Relaxation method using the Absorbing Boundary Condition taken as an approximation of the Transparent Operator for the interface condition. We must add terms to enrich the equations associated to the Absorbing Boundary Condition and compute them with a cumulative process. Numerical examples are used to show the performance of this Domain Decomposition Method.
\end{abstract}

Keywords: Absorbing Boundary Conditions, Domain Decomposition Method, Transparent Operator, Stratified Dispersive Wave Model.

\section{Introduction}

In the context of problems based on the wave equation, the Absorbing Boundary Conditions (ABC) of Engquist and Majda [1] applied on the boundaries of truncated domain simulate the presence of the infinite complementary domain. There must be approached in the case of domains in two or three dimensions. Applied to one of the boundaries of the computational domain, this allows to the waves approaching the boundary to go out properly of the simulation domain. The order of approximation of the $\mathrm{ABC}$ and the incidence angle of the waves arriving on these boundaries play on the quality output of these waves. Higdon ([3], 4]) proposed an approximate ABC to treat wave output with different incidence angles. Collino [5], Grote and Keller [7], Hagstrom and Hariharan [8], Guddati and Tassoulas [9], Givoli and Neta [10] and Hagstrom and Warburton [11, by the use of auxiliary variables, proposed a way to write the boundary condition using only order one numerical derivatives. The Hagstrom and Warburton's ABC corresponds to a double form of the Higdon Boundary Condition.

Email address: olivier.wilk@cnam.fr (O. Wilk) 
It allows a better numerical stability for longer periods of simulation than Givoli and Neta's ABC. We choose here to use the Hagstrom and Warburton's ABC (HW-ABC). Other methods, for example such as the Perfectly Matched Layer (PML) method devised by Bérenger [6], can be use. Givoli proposed a review 2o of others non-reflecting boundary conditions [12].

This boundary condition has been successfully applied to different models of waves, the wave equation, the dispersive wave equation, convected and for layered media or for continuous wave speed variation ([13], [14]).

We apply it to a Stratified Dispersive Wave Model (section 2). A constraint due to the coupling is required ([13]).

The model's computation has a significant cost when the simulations are performed with a full size domain. So these can be reduced by parallel computing, Domain Decomposition Methods (Schur [17, Multigrid [18], FETI [20] methods, ...). In our case, we note that the $\mathrm{ABC}$ is useful to determine the boundaryinterface condition to connect the different subdomains with the Schwarz Waveform Relaxation (SWR) "Domain Decomposition" Method (15, [19, 21, 22]). The works of Gander, Halpern ([23], [25]) and Nataf [24] emphasize very clearly the advantage of using the transparent operator to build an optimal interface condition. This transparent operator is the exact ABC. Here we choose to approach this operator by the HW-ABC (section 3). The convergence rate is done in the section 4 .

We illustrate these works with numerical applications in the last section of this article.

\section{The Absorbing Boundary Condition of Hagstrom and Warburton applied to a Stratified Dispersive Wave Model}

We present here the problem-model and the ABC of Hagstrom and Warburton applied to a stratified material.

The Dispersive Wave Model in the domain $\Omega(c, \alpha \in \mathbb{R}, t \in[0, T]$, figure 1):

$$
\left\{\begin{array}{l}
\left(\partial_{t}^{2}-c^{2} \Delta+\alpha^{2}\right) u=0 \text { in } \Omega, \\
\partial_{\nu} u=0 \text { on } \Gamma_{t} \cup \Gamma_{b}, \\
\text { with ABC on } \Gamma_{l} \cup \Gamma_{r}, \\
\text { and initial conditions, }
\end{array}\right.
$$

uses Absorbing Boundary Conditions on the left boundary $\Gamma_{l}$ and the right boundary $\Gamma_{r}$ (for our example), the boundaries of a truncated domain, to simulate the presence of the infinite complementary domain. These can be defined using the transport equation reported here at the boundary of the outer normal $\nu$ of the domain $\Omega$ [1]:

$$
\left(\partial_{t}+c \partial_{\nu}\right) u=0 \text { on } \Gamma_{l} \cup \Gamma_{r} .
$$

To one-dimensional non-dispersive wave model, the equation (2) is the exact condition. With two or three space dimensions or with a dispersive model, it is 


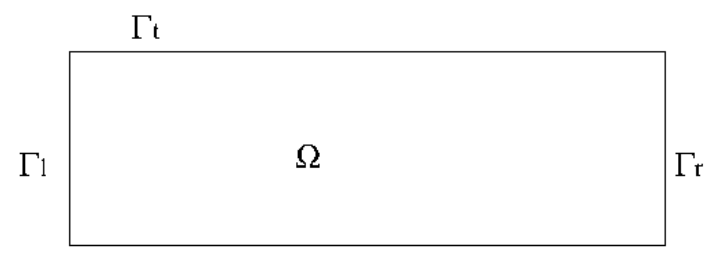

$\Gamma \mathrm{b}$

Figure 1: The domain and the boundaries.

an approximation, usually the first order approximation. We must use an other (better) condition. Different non-reflecting boundary conditions exist. The Higdon condition ([3], 4]), Collino condition [5, Grote and Keller condition [7, Hagstrom and Hariharan condition 8], Guddati and Tassoulas condition [9], Givoli and Neta condition [10, Hagstrom and Warburton condition [11 and also the Perfectly Matched Layer (PML) method devised by Bérenger [6] can be used to better results. Here, we apply the High-Order Absorbing Boundary Condition of Hagstrom and Warburton (HW-ABC) (with the new auxiliary variables $\left.\phi_{i}(i=1, n+1)\right)$ :

$$
\begin{cases}\text { on } \Gamma_{l} \cup \Gamma_{r}: & \\ \left(a_{0} \partial_{t}+c \partial_{\nu}\right) u & =a_{0} \partial_{t} \phi_{1}, \\ \left(a_{i} \partial_{t}+c \partial_{\nu}\right) \phi_{i} & =\left(a_{i} \partial_{t}-c \partial_{\nu}\right) \phi_{i+1}, \quad \forall i=1, n, \\ \phi_{n+1} & =0 .\end{cases}
$$

Each auxiliary variable $\phi_{i}$ solves, by the linearity of the operators, the same model as $u$. Fortunately, it is possible to reduce the size of each auxiliary problem [14]. The boundary condition corresponds to a " $2 n+1 "$ order of the boundary condition of Higdon, ie:

$$
\left(a_{0} \partial_{t}+c \partial_{\nu}\right) \prod_{i=1}^{n}\left(a_{i} \partial_{t}+c \partial_{\nu}\right)^{2} u=0 \text { on } \Gamma_{l} \cup \Gamma_{r} .
$$

As shown in 13, this condition can be used on a Stratified Dispersive Wave Model (figure 2):

$$
\left\{\begin{array}{l}
\left(\partial_{t}^{2}-c_{1}^{2} \Delta+\alpha_{1}^{2}\right) u_{1}=0 \text { in } \Omega_{1}, \partial_{\nu_{1}} u=0 \text { on } \Gamma_{t}, \\
\left(\partial_{t}^{2}-c_{2}^{2} \Delta+\alpha_{2}^{2}\right) u_{2}=0 \text { in } \Omega_{2}, \partial_{\nu_{2}} u=0 \text { on } \Gamma_{b},
\end{array}\right.
$$

with a compatibility constraint for the " $a_{i}$ " coefficients at the interface between the two domains. This can be written:

$$
\frac{c_{1}}{a_{1, i}}=\frac{c_{2}}{a_{2, i}}, \forall i=0, n,
$$

with $a_{1, i}$ and $a_{2, i}$ the coefficients associated respectively to the domain with the $c_{1}$ coefficient and the domain with the $c_{2}$ coefficient for the ABC conditions on so the boundaries $\Gamma_{l j}$ and $\Gamma_{r j}(j=1,2)$. So with these constraints, the boundary conditions are continuous at the intersection between the boundaries $\Gamma_{l j}$ or $\Gamma_{r j}$ and the interface $\Gamma_{12}$ between the two different materials. 


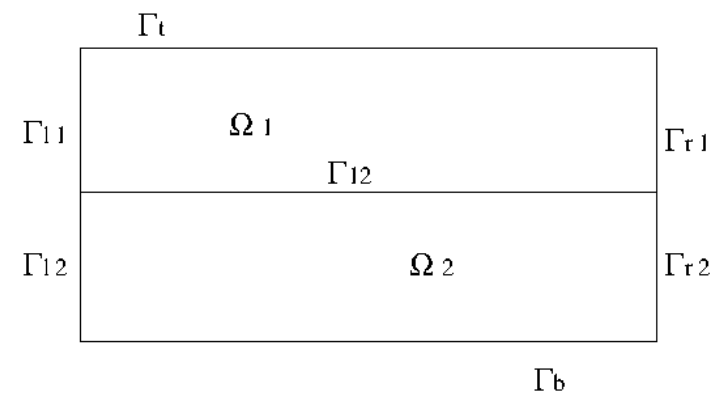

Figure 2: The domains and the boundaries for the stratified problem.

So in each domain, the problem can be written with the reduced $\phi_{i}$ problems (with $\phi^{0}=u=u_{j}, a_{i}=a_{j, i}$ for the $j$ domain $(j=1,2)$ ):

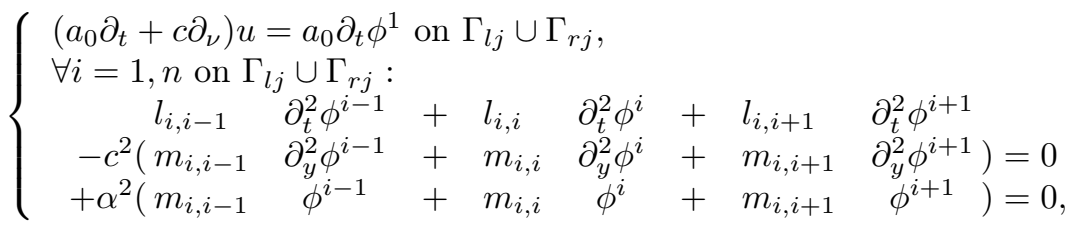

with the zero initial conditions, the compatibility conditions 6 and:

$$
\left\{\begin{array}{ccccccc}
i=1: & & & & & \\
l_{1,0}= & 2 a_{1}\left(1-a_{0}^{2}\right), & m_{1,0}= & 2 a_{1}, & & \\
l_{1,1}= & a_{0}\left(1+2 a_{0} a_{1}+a_{1}^{2}\right), & m_{1,1}= & a_{0}, & & \\
l_{1,2}= & a_{0}\left(1-a_{1}^{2}\right), & m_{1,2}= & a_{0}, & & \\
i>1: & & a_{i}\left(1-a_{i-1}^{2}\right), & & & & \\
l_{i, i-1} & = & & & & & \\
l_{i, i} & = & a_{i}\left(1+a_{i-1}^{2}\right)+a_{i-1}\left(1+a_{i}^{2}\right), & m_{i, i} & = & a_{i-1}+a_{i}, \\
l_{i, i+1} & = & a_{i-1}\left(1-a_{i}^{2}\right), & & m_{i, i+1} & = & a_{i-1} .
\end{array}\right.
$$

\section{The Absorbing Boundary Condition of Hagstrom and Warburton applied to a DDM Stratified Dispersive Wave Model}

We present the elements allowing us to perform a domain decomposition method using the HW-ABC. We start with the Classical Schwarz Waveform Relaxation ([15]) assisted by an interface condition. We choose to use the transparent operator approximated here by the $\mathrm{HW}-\mathrm{ABC}$ at the boundary-interface between subdomains.

\subsection{Schwarz Waveform Relaxation and the interface boundary condition}

We present the parallel version ([16]) of the Schwarz Waveform Relaxation algorithm (SWR) with overlapping subdomains on the wave equation (with $\left.\square_{c} u=\partial_{t} u^{2}-c^{2} \Delta u\right)$, using the Dirichlet interface condition and the error variables " $u_{i}^{p}-u_{i}^{p-1 "}(i=1,2)$ that we note abusively thereafter $u_{i}^{p}$ (figure 3 : 


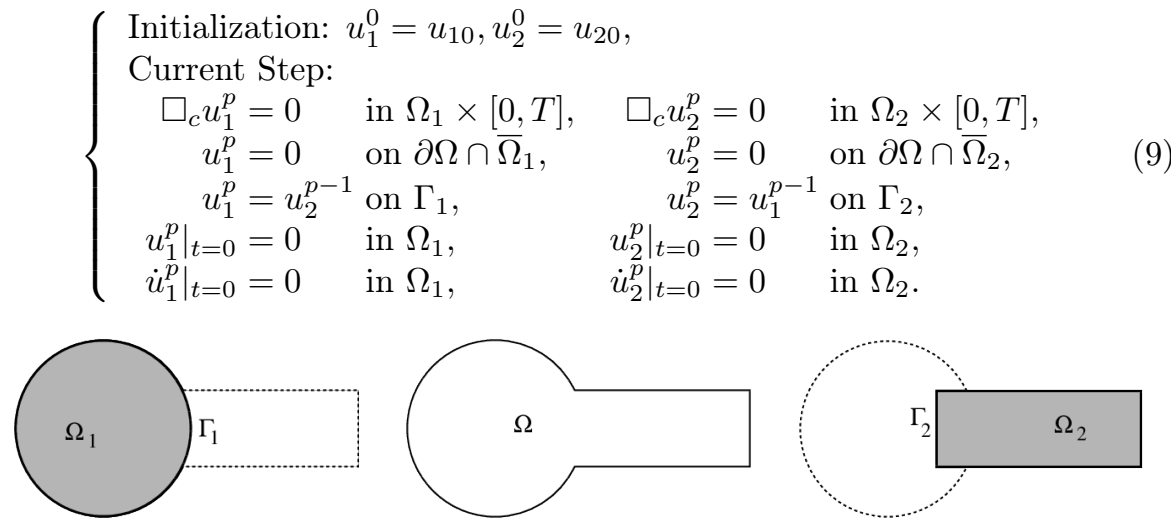

Figure 3: A domain decomposition associated with the problem 9 .

Noting $\delta$ the overlap distance domain (between $\Gamma_{1}$ and $\Gamma_{2}$ ), $p$ the number of iterations, the convergence of such process checks [25]:

$$
p>\frac{T c}{\delta} .
$$

This convergence can be improved by changing the interface condition. We write it in a generic way:

$$
B_{i} u_{i}^{p}=B_{i} u_{j}^{p-1} \text { on } \Gamma_{i}, i=1,2 .
$$

We may note here that all the second members, apart from the interface conditions, are equal to zero. Thus, it would be interesting to have an operator $B_{i}, i=1,2$ as (for $u_{i}^{p-1}$ non-zero, $i=1,2$ ):

$$
\left\{\begin{array} { l } 
{ B _ { 1 } u _ { 2 } ^ { p - 1 } \neq 0 } \\
{ B _ { 2 } u _ { 1 } ^ { p - 1 } \neq 0 }
\end{array} \Rightarrow \left\{\begin{array}{l}
B_{1} u_{2}^{p}=0 \\
B_{2} u_{1}^{p}=0
\end{array},\right.\right.
$$

so we will get $u_{i}^{p}$ equal to zero $(i=1,2)$. Gander, Halpern and Nataf [24] proved that such operator exists in particular when there is non overlapping domains. We consider here the "Dirichlet to Neumann" operator $\left(\nu_{1}\right.$ the outward normal on $\Gamma_{1}$ a part of the boundary of the domain $\Omega_{1}$ ):

$$
\operatorname{DtN}_{1}(v): v \text { on } \Gamma_{1} \mapsto \frac{\partial u}{\partial \nu_{1}} \text { on } \Gamma_{1},
$$

associated with the problem:

$$
\left\{\begin{array}{lll}
\square_{c} u_{1}=0 & \text { in } \Omega_{1} \times[0, T], \\
u_{1}=0 & \text { on } \partial \Omega \cap \bar{\Omega}_{1}, \\
u_{1}=v & \text { on } \Gamma_{1}, \\
\left.u_{1}\right|_{t=0}=0 & ,\left.\dot{u}_{1}\right|_{t=0}=0 \text { in } \Omega_{1} .
\end{array}\right.
$$

With $\Gamma_{2}$ equal to $\Gamma_{1}$ (non overlapping), we can write (flow conservation, $u_{1}=v$ on $\Gamma_{1}$ and $\nu_{2}$ the outward normal on $\Gamma_{2}$ of $\Omega_{2}$ ):

$$
\frac{\partial u_{1}}{\partial \nu_{2}}=-\frac{\partial u_{1}}{\partial \nu_{1}}=-D t N_{1}(v)=-D t N_{1}\left(u_{1}\right) .
$$


So we get the operator $B_{2}$ such that if $u_{1}$ satisfies (14) and this for all $v$ :

$$
B_{2}\left(u_{1}\right)=\frac{\partial u_{1}}{\partial \nu_{2}}+\operatorname{Dt} N_{1}\left(u_{1}\right)=0 \text { on } \Gamma_{2} .
$$

We proceed in the same way to get $B_{1}$. Both called transparent operators are used to verify (12). They allow to converge the Schwarz algorithm (9) only with two iterations [24]. This is known as optimal convergence.

This type of operator is the exact $\mathrm{ABC}$ [24]. But it is too expensive to use it. We prefer a less expensive approximation. That is why we want to use a interface condition built using the $\mathrm{HW}-\mathrm{ABC}$ to process here.

\subsection{The interface condition with the Hagstrom and Warburton absorbing bound-} ary condition

The interface condition using the HW-ABC can be implemented easily using the previous formalism, choosing $B_{1}$ in the proposed form (4). Thus the interface condition (11) becomes (for $i=1$ ):

$$
\left[\prod_{j=1}^{n}\left(a_{j} \partial_{t}+c \partial_{\nu}\right)^{2}\right]\left(a_{0} \partial_{t}+c \partial_{\nu}\right) u_{1}^{p}=\left[\prod_{j=1}^{n}\left(a_{j} \partial_{t}+c \partial_{\nu}\right)^{2}\right]\left(a_{0} \partial_{t}+c \partial_{\nu}\right) u_{2}^{p-1},
$$

or:

$$
\left[\prod_{j=1}^{n}\left(a_{j} \partial_{t}+c \partial_{\nu}\right)^{2}\right]\left(a_{0} \partial_{t}+c \partial_{\nu}\right)\left(u_{1}^{p}-u_{2}^{p-1}\right)=0 \text { on } \Gamma_{1} .
$$

With the auxiliary variable form of HW-ABC, we can write $\left(U_{i}^{T}=\left(u_{i}=\right.\right.$ $\left.\left.\phi_{i, 0} ; \phi_{i, 1} ; \ldots ; \phi_{i, n}\right), i=1,2\right)$ :

$$
\mathcal{B}_{i} U_{i}^{p}=0 \Leftrightarrow\left\{\begin{array}{l}
\text { on } \Gamma_{i}: \\
\left(a_{0} \partial_{t}+c \partial_{\nu_{i}}\right) u_{i}^{p}=a_{0} \partial_{t} \phi_{i, 1}^{p}, \\
\forall k=1, n: \\
\left(a_{k} \partial_{t}+c \partial_{\nu_{i}}\right) \phi_{i, k}^{p}=\left(a_{k} \partial_{t}-c \partial_{\nu_{i}}\right) \phi_{i, k+1}^{p}, \\
\phi_{i, n+1}^{p}=0 .
\end{array}\right.
$$

So on each DDM interface, we want to use the interface condition:

$$
\mathcal{B}_{i} U_{i}^{p}=\mathcal{B}_{i} U_{j}^{p-1}, i, j=1,2 \text { and } i \neq j .
$$

The first equation of the previous condition is applied with this form. But for the others equations, we prefer to use the classical practical form of the HW-ABC. So we keep the first equation:

$$
\left(a_{0} \partial_{t}+c \partial_{\nu_{i}}\right)\left(u_{i}^{p}-u_{j}^{p-1}\right)-a_{0} \partial_{t}\left(\phi_{i, 1}^{p}-\phi_{j, 1}^{p-1}\right)=0 \text { on } \Gamma_{i} .
$$

For the others with the classical calculation $\left(\right.$ on $\left.\Gamma_{i}\right)$ :

$$
\left[a_{k-1}\left(a_{k} \partial_{t}+c \partial_{\nu_{i}}\right) \mathcal{B}_{i, k}+2 a_{k}\left(a_{k-1} \partial_{t}-c \partial_{\nu_{i}}\right) \mathcal{B}_{i, k-1}\right]\left(U_{i}^{p}-U_{j}^{p-1}\right)=0,
$$


the difference $\left(U_{i}^{p}-U_{j}^{p-1}\right)$ verifies $(\forall k=1, n)$ :

$$
\begin{array}{ll} 
& {\left[l_{k, k-1} \partial_{t}^{2}+m_{k, k-1}\left(\alpha^{2}-c^{2} \partial_{y}^{2}\right)\right]\left(\phi_{i, k-1}^{p}-\phi_{j, k-1}^{p-1}\right)} \\
+ & {\left[l_{k, k} \partial_{t}^{2}+m_{k, k}\left(\alpha^{2}-c^{2} \partial_{y}^{2}\right)\right]\left(\phi_{i, k}^{p}-\phi_{j, k}^{p-1}\right)} \\
+ & {\left[l_{k, k+1} \partial_{t}^{2}+m_{k, k+1}\left(\alpha^{2}-c^{2} \partial_{y}^{2}\right)\right]\left(\phi_{i, k+1}^{p}-\phi_{j, k+1}^{p-1}\right)=0} \\
\Leftrightarrow & \mathcal{C}_{i}\left(U_{i}^{p}-U_{j}^{p-1}\right)=0 \text { on } \Gamma_{i} .
\end{array}
$$

Of course, the $\mathcal{C}_{i}$ operators are equal (with the same coefficient families $l_{i, j}$ and $m_{i, j}$ in the two subdomains):

$$
\mathcal{C}_{1}=\mathcal{C}_{2}
$$

As we begin the SWR iterative process with the HW-ABC equal to zero:

$$
\mathcal{C}_{i} U_{i}^{0}=0 \text { on } \Gamma_{i}, \forall i=1,2 .
$$

So, we keep that at each iteration:

$$
\mathcal{C}_{i} U_{i}^{p}=0 \text { on } \Gamma_{i}, \forall i=1,2 \text { and } \forall p \in \mathbb{N} .
$$

This allows to write a useful form for numerical simulation with the new variables $\lambda_{1}^{p-1}$ and $\lambda_{2}^{p-1}$ :

$$
\left\{\begin{array}{l}
\left\{\begin{array}{l}
\text { on } \Gamma_{1}: \\
\left(a_{0} \partial_{t}+c \partial_{\nu_{1}}\right) u_{1}^{p}=a_{0} \partial_{t} \phi_{1,1}^{p}+\lambda_{1}^{p-1} \\
\left(a_{j} \partial_{t}+c \partial_{\nu_{1}}\right) \phi_{1, j}^{p}=\left(a_{j} \partial_{t}-c \partial_{\nu_{1}}\right) \phi_{1, j+1}^{p} \\
\phi_{1, n+1}^{p}=0
\end{array}, \forall j=1, n,\right. \\
\left\{\begin{array}{l}
\text { on } \Gamma_{2}: \\
\left(a_{0} \partial_{t}+c \partial_{\nu_{2}}\right) u_{2}^{p}=a_{0} \partial_{t} \phi_{2,1}^{p}+\lambda_{2}^{p-1} \\
\left(a_{j} \partial_{t}+c \partial_{\nu_{2}}\right) \phi_{2, j}^{p}=\left(a_{j} \partial_{t}-c \partial_{\nu_{2}}\right) \phi_{2, j+1}^{p} \\
\phi_{1, n+1}^{p}=0,
\end{array}, \forall j=1, n,\right. \\
\text { with } \begin{array}{l}
\lambda_{1}^{p-1}=\left(a_{0} \partial_{t}+c \partial_{\nu_{1}}\right) u_{2}^{p-1}-a_{0} \partial_{t} \phi_{2,1}^{p-1} \\
\lambda_{2}^{p-1}=\left(a_{0} \partial_{t}+c \partial_{\nu_{2}}\right) u_{1}^{p-1}-a_{0} \partial_{t} \phi_{1,1}^{p-1}
\end{array}
\end{array}\right.
$$

So we write:

$$
\left\{\begin{array}{l}
\lambda_{1}^{p}=-\left(a_{0} \partial_{t}+c \partial_{\nu_{2}}\right) u_{2}^{p}+2 a_{0} \partial_{t} u_{2}^{p}-a_{0} \partial_{t} \phi_{2,1}^{p-1}, \\
\lambda_{2}^{p}=-\left(a_{0} \partial_{t}+c \partial_{\nu_{1}}\right) u_{1}^{p}+2 a_{0} \partial_{t} u_{1}^{p}-a_{0} \partial_{t} \phi_{1,1}^{p-1},
\end{array}\right.
$$

and with 27), we get the specific cumulative process to build the $\lambda$ variables:

$$
\left\{\begin{array}{l}
\lambda_{1}^{p}=-\lambda_{2}^{p-1}+2 a_{0} \partial_{t}\left(u_{2}^{p}-\phi_{2,1}^{p}\right), \\
\lambda_{2}^{p}=-\lambda_{1}^{p-1}+2 a_{0} \partial_{t}\left(u_{1}^{p}-\phi_{1,1}^{p}\right) \\
\text { with } \lambda_{i}^{0}=0, \forall i=1,2 .
\end{array}\right.
$$

For our DDM problem, the HW-ABC is enhanced by the $\lambda$ variables $\left(\lambda_{1}^{p-1}\right.$ and $\lambda_{2}^{p-1}$ ) computed with the classical state solution and just the first auxiliary 
variable of the neighbouring subdomain without overlapping.

Remark: The HW-ABC can be written to work with evanescent waves [13. This can be done with other $m$ new auxiliary variables useful to write the new part of the boundary condition with $m$ new parameters $\sigma_{k}$ :

$$
\left(\sigma_{k}+\partial_{\nu}\right) \phi_{n+k}=\left(\sigma_{k}-\partial_{\nu}\right) \phi_{n+k+1}, \forall k=1, m, \text { and } \phi_{n+m+1}=0 .
$$

So the last auxiliary variable $\phi_{n+1}$ (associated to the propagating waves) is no longer set to zero.

With a similar way, the last equations can be transformed to get reduced problems just on the boundary and each operator are always equal in the two subdomains. So we keep the previous result with the inclusion of evanescent modes.

so We can get more precisions in [13.

\section{Convergence rate}

We compute the convergence rate of the following HW-ABC DDM error problem (error variables $v_{l}^{p}=u_{l}^{p}-u, \forall l=1,2, \delta$ measure of overlapping distance):

$$
\left\{\begin{array}{l}
\left(\partial_{t}^{2}-c^{2}\left(\partial_{x}^{2}+\partial_{y}^{2}\right)+\alpha^{2}\right) v_{1}^{p}=0 \text { for } x<\delta, \square_{1} v_{1}^{p}=\square_{1} v_{2}^{p-1} \text { on } x=\delta, \\
\left(\partial_{t}^{2}-c^{2}\left(\partial_{x}^{2}+\partial_{y}^{2}\right)+\alpha^{2}\right) v_{2}^{p}=0 \text { for } x>0, \square_{2} v_{2}^{p}=\square_{2} v_{1}^{p-1} \text { on } x=0, \\
\text { with } \square_{l}=\left[\prod_{j=1}^{n}\left(a_{j} \partial_{t}+c \partial_{\nu_{l}}\right)^{2}\right]\left(a_{0} \partial_{t}+c \partial_{\nu_{l}}\right), \forall l=1,2 .
\end{array}\right.
$$

With a Fourier transform in $y$ and $t$ :

$$
\hat{v}(x, k, \omega)=\frac{1}{2 \pi} \int_{\mathbb{R}^{2}} v(x, y, t) e^{-i(\omega t+k y)} d y d t,
$$

applied to the previous DDM problem, we obtain:

$$
\left\{\begin{array}{l}
\left(-c^{2} \partial_{x}^{2}+c^{2} k^{2}+\alpha^{2}-\omega^{2}\right) v_{1}^{p}=0 \text { for } x<\delta, \hat{\square}_{1} v_{1}^{p}=\hat{\square}_{1} v_{2}^{p-1} \text { on } x=\delta, \\
\left(-c^{2} \partial_{x}^{2}+c^{2} k^{2}+\alpha^{2}-\omega^{2}\right) v_{2}^{p}=0 \text { for } x>0, \hat{\square}_{2} v_{2}^{p}=\hat{\square}_{2} v_{1}^{p-1} \text { on } x=0, \\
\text { with } \hat{\square}_{l}=\left[\prod_{j=1}^{n}\left(i a_{j} \omega+c \partial_{\nu_{l}}\right)^{2}\right]\left(i a_{0} \omega+c \partial_{\nu_{l}}\right), \forall l=1,2 .
\end{array}\right.
$$

With the Sommerfeld condition, the left and right solutions are:

$$
\begin{aligned}
& \hat{v}_{1}^{p}=A_{1}^{p} e^{\xi(x-\delta)}, \hat{v}_{2}^{p}=A_{2}^{p} e^{-\xi x} \\
& \text { with } \xi=\left\{\begin{array}{l}
\sqrt{k^{2}+\frac{\alpha^{2}}{c^{2}}-\frac{\omega^{2}}{c^{2}}} \text { if } k^{2}+\frac{\alpha^{2}}{c^{2}} \geq \frac{\omega^{2}}{c^{2}} \text { (evanescent waves), } \\
i \sqrt{\frac{\omega^{2}}{c^{2}}-k^{2}-\frac{\alpha^{2}}{c^{2}}} \text { if } k^{2}+\frac{\alpha^{2}}{c^{2}}<\frac{\omega^{2}}{c^{2}} \text { (propagative waves). }
\end{array}\right.
\end{aligned}
$$


The constants $A_{1}^{p}$ and $A_{2}^{p}$ can be determined with the Fourier transform of the $\mathrm{HW}-\mathrm{ABC}$ interface condition and the previous values $A_{1}^{p-1}$ and $A_{2}^{p-1}$. So the convergence rate $\tau$ is given by:

$$
\tau=\left|\frac{A_{1}^{p}}{A_{1}^{p-2}}\right|^{\frac{1}{2}}=\left|\frac{A_{2}^{p}}{A_{2}^{p-2}}\right|^{\frac{1}{2}}=\frac{\left(i a_{0} \omega-c \xi\right) \prod_{j=1}^{n}\left(i a_{j} \omega-c \xi\right)^{2}}{\left(i a_{0} \omega+c \xi\right) \prod_{j=1}^{n}\left(i a_{j} \omega+c \xi\right)^{2}} e^{-\xi \delta}
$$

The figure 4 shows some examples for a stratified case of the first monomial of
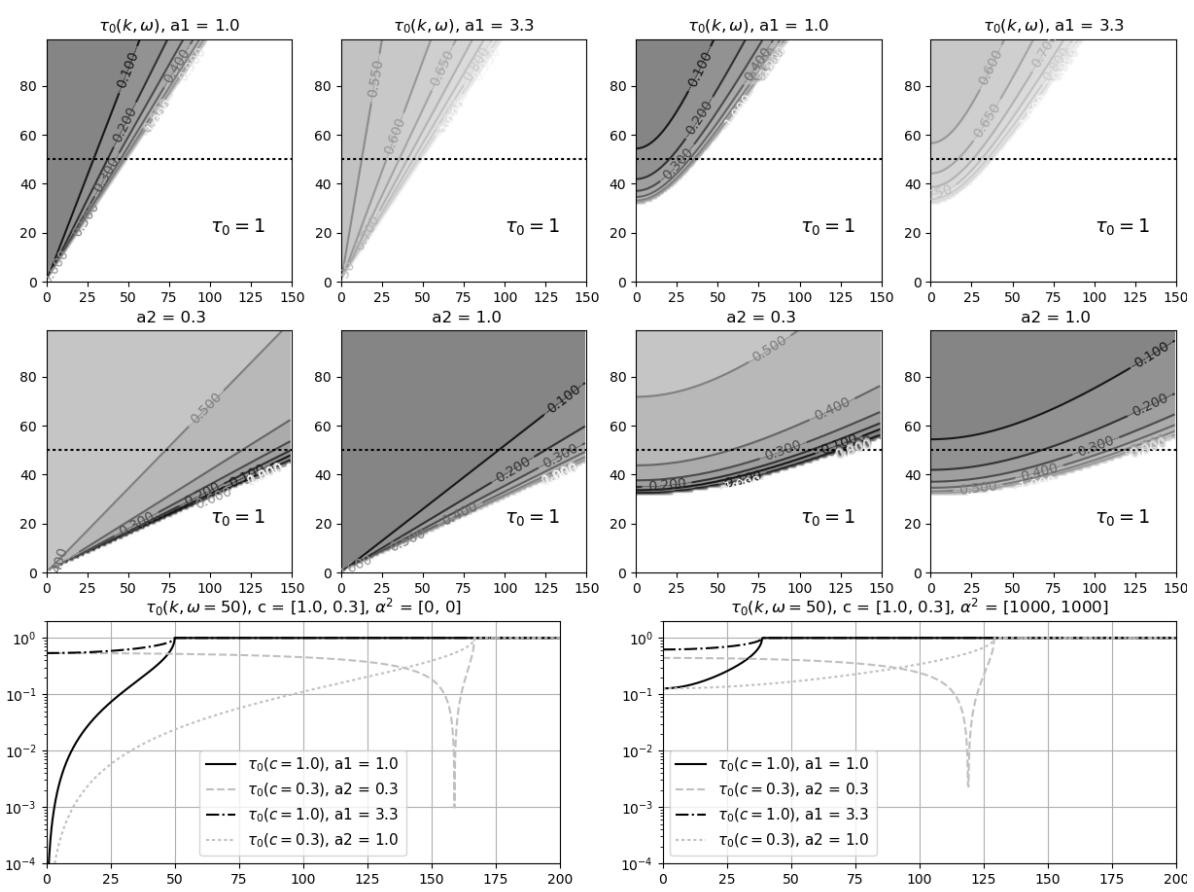

Figure 4: First monomial 36 of $\tau$, 35 with different values of $a_{0}$ for a stratified case: for the first line, $c$ is equal to one in the domain and 0.3 for the second line, the left part is for a none dispersive case and the right for a dispersive case. The coefficients $a 1$ (for the domain with $c$ equal to one), and $a 2$ (for $c$ equal to 0.3 ) verify the compatibility equation 6 . The last line shows cuts of the monomial associated to the dotted lines of the two top graph-lines.

$\tau$ (35) with different values of $a_{0}$ with the propagatives mode:

$$
\tau_{0}(\omega, k)=\frac{\left(i a_{0} \omega-c \xi\right)}{\left(i a_{0} \omega+c \xi\right)} .
$$

The stratified case example corresponds to a top domain with $c$ equal to one and a bottom domain with $c$ equal to 0.3 . We use the compatibility equation (6) to compute the coefficient $a_{0}$ for the top and the bottom domains. To get the numerical values of $\tau$, we combine (multiply) different monomials as the first monomial of the figure 4. The result is shown in the figure 5.

As illustrated by the figures 4 and 5 , the convergence rate is always less than one when the solution is composed by the propagative waves. If the wave number $k$ is greater than the limit between propagative and evanescent waves, the evanescent waves part gives a convergence rate equal to one. But it is not 

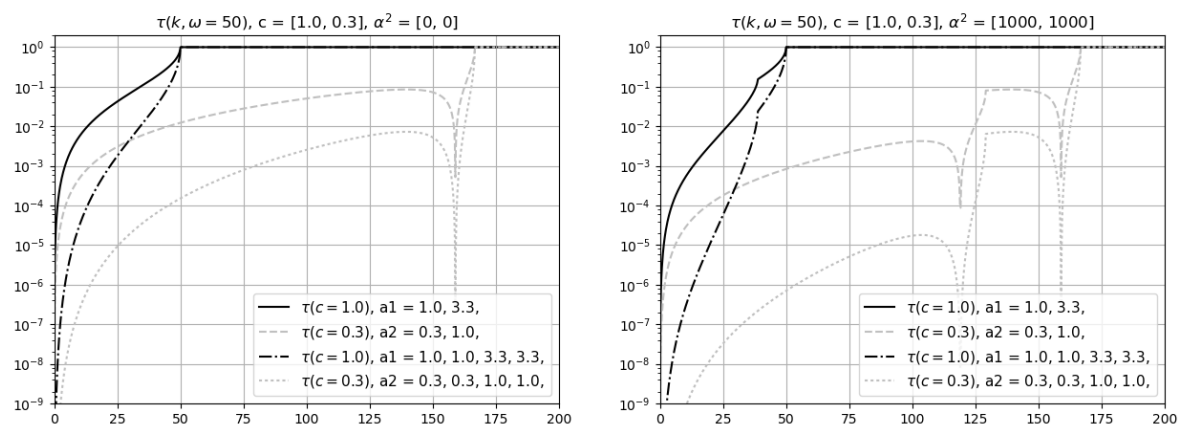

Figure 5: Examples of the convergence rate $\tau$ associated to the figure 4 for different sets of the coefficients $a_{i}$.

a practical problem when the principal problem is composed by the propagative waves, the evanescent waves are sufficiently small. An overlapping is not required, as we will see with the numerical applications (next section). We can note that if we want to use an overlapping, it is a more expensive choice. For this case, we must compute the first auxiliary variable $\phi_{1}$ in the domain, not just in the boundary.

Remark: If the ABC-boundary is near a source or a boundary, the evanescent waves may be more significant. In theses cases, we can complete the $\mathrm{ABC}$ condition with a part designed to evanescent waves. So theoretically, we can avoid to use an overlapping for the SWR problem. To absorb the evanescent waves on the boundary $\Gamma$, we can employ $m$ new auxiliary variables $\phi$ as following [13:

$$
\left\{\begin{array}{l}
\text { on } \Gamma: \\
\left(a_{0} \partial_{t}+c \partial_{\nu}\right) u=a_{0} \partial_{t} \phi_{1} \\
\left(a_{k} \partial_{t}+c \partial_{\nu}\right) \phi_{k}=\left(a_{k} \partial_{t}-c \partial_{\nu}\right) \phi_{k+1}, \forall k=1, n \\
\left(\sigma_{k}+\partial_{\nu}\right) \phi_{n+k}=\left(\sigma_{k}-\partial_{\nu}\right) \phi_{n+k+1}, \forall k=1, m, \\
\phi_{n+m+1}=0
\end{array}\right.
$$

The $k$ th monomial associated to the evanescent- $\mathrm{ABC}$ of the convergence rate of the SWR problem is:

$$
\frac{\left(\sigma_{k}-\xi\right)}{\left(\sigma_{k}+\xi\right)}
$$

So with $\sigma_{k}$ equal (or sufficiently near) to $\xi$, we can get the convergence less than one without overlapping.

\section{Numerical applications}

For the numerical applications, we work with a bidimensional domain. The reference domain (figure 6) is $\left[-l_{x}, l_{x}\right] \times[-0.3,0]$ (with different values for the length $l_{x}$ ). For the space discretization of the domain, we use the Finite Element (FE) formulation with an uniform mesh with square elements. We use the standard spatial Galerkin FE discretization with the polynomial functions of Lagrange of order two.

For the time discretization, we choose the implicit version of the Newmark 


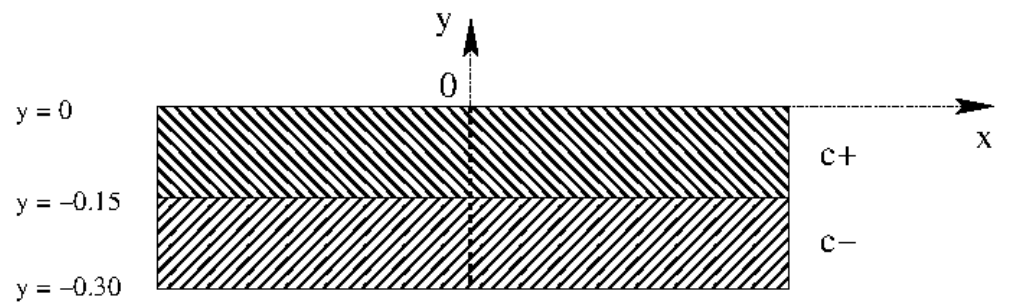

Figure 6: The computational domain for a stratified case $c^{-} \leq c^{+}$.

scheme $(\gamma=0.5, \beta=0.25)$ to choose different time steps designed for fast and slow domains. We use two different time steps, a small time step $\delta t$ equal to $\sqrt{2} / 2$ of the minimum size $h$ of the space element divided by $c^{+}$the maximum of $c$ (always equal to one for our applications) and a big time step equal to the small time step divided by $c^{-}$the minimum of $c$ (less than one for the stratified cases).

For the initial condition, we use a gaussian function with the initial velocity equal to zero:

$$
u_{0}(x, y)=e^{-\frac{\left(x-x_{0}\right)^{2}}{2 \sigma^{2}}}, \text { with } \sigma^{2}=10^{-3}, x_{0}=-0.15 .
$$

This signal is composed of different $x$ space frequencies as shown in figure 7 . For the stratified cases with this initial condition, an important part of the solution is composed of propagative waves with different incidence angles near the ABC boundaries for positive times. The initial plane waves have been transformed. And at the interface between the two different materials, we have local solutions 27 .
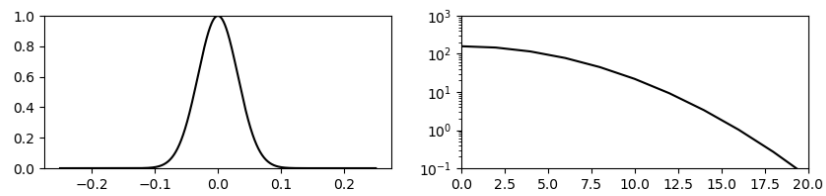

Figure 7: The initial condition $e^{-\frac{x^{2}}{2 \sigma^{2}}}\left(\right.$ left, $\left.x_{0}=0\right)$ with the associated frequencies (right).

We compute the solution $u$ in the domain $\left[-l_{x}, 0\right] \times[-0.3,0]$ for the ABC tests and in the two subdomains $\left[-l_{x}, 0\right] \times[-0.3,0]$ (the left subdomain) and $\left[0, l_{x}\right] \times[-0.3,0]$ (the right subdomain) for the SWR tests. In the reference domain $\left[-l_{x}, l_{x}\right] \times[-0.3,0]$, we get the reference solution $u_{r e f}$. We can compare the solutions with the $L_{2}$ difference at each time $t$ in the domain $\Omega_{u}$ (usually $\left[-l_{x 0}, 0\right] \times[-0.3,0]$ for the ABC tests and $\left[-l_{x 0}, 0\right] \times[-0.3,0] \cup\left[0, l_{x 0}\right] \times[-0.3,0]$ for the DDM tests $\left.\left(l_{x 0}=0.6<l_{x}\right)\right)$ or on a boundary or on the interface between two materials:

$$
\frac{\sqrt{\frac{1}{\operatorname{mes}\left(\Omega_{u}\right)} \int_{\Omega_{u}}\left|u-u_{r e f}\right|^{2}}}{\max \left(\left|u_{0}\right|\right)} \text { with } \max \left(\left|u_{0}\right|\right)=1 \text {. }
$$

We use two small time steps $\delta t$ near to $0.007,0.0035 s$ respectively for the space steps $h$ equal to $0.01,0.005 \mathrm{~m}$ with different values of $c_{-}$equal to $1,0.3,0.1 \mathrm{~m} . \mathrm{s}^{-1}$ 


\begin{tabular}{|c|c|c|c|c|c|c|c|}
\hline HW-ABC & $c_{+}$ & $c_{-}$ & $\alpha^{2}$ & $T$ & $l_{x}$ & $\delta t$ & $h$ \\
\hline & 1 & 1 & 100 & $0.5 s$ & $l_{x 0}$ & small & $h \& h / 2$ \\
\hline & 1 & 0.3 & 100 & $3 \times 0.5 s$ & $3 \times l_{x 0}$ & small & $h \& h / 2$ \\
\hline HW-SWR & $c_{+}$ & $c_{-}$ & $\alpha^{2}$ & $T$ & $l_{x}$ & $\delta t$ & $h$ \\
\hline & 1 & 0.3 & $0 \& 100$ & $3 \times 0.5 s$ & $3 \times l_{x 0}$ & small \& big & $h \& h / 2$ \\
\hline & 1 & 0.1 & $0 \& 100$ & $10 \times 0.5 s$ & $10 \times l_{x 0}$ & small \& big & $h \& h / 2$ \\
\hline
\end{tabular}

Table 1: The different used configurations to test only the HW-ABC (HW-ABC, section 5.1 and the SWR process using the HW-ABC (HW-SWR, section 5.2.

\begin{tabular}{|c|l|l|}
\hline order & top domain $c_{+}$ & bottom domain $c_{-}$ \\
\hline 1 & 1 & $c_{-+}$ \\
\hline 2 & $1, c_{+-}$ & $c_{-+}, 1$ \\
\hline 4 & $1,1, c_{+-}, c_{+-}$ & $c_{-+}, c_{-+}, 1,1$ \\
\hline 8 & $1,1,1,1, c_{+-}, c_{+-}, c_{+-}, c_{+-}$ & $c_{-+}, c_{-+}, c_{-+}, c_{-+}, 1,1,1,1$ \\
\hline 16 & $1,1,1,1,1,1,1,1, c_{+-}, \ldots$ & $\ldots$ \\
\hline 32 & $\ldots$ & $\ldots$ \\
\hline
\end{tabular}

Table 2: HW-ABC coefficients for the top and the bottom domains for different orders using the compatibility condition $[6$. For the orders 16 and 32, we use the same way to determine the coefficients (with $c_{+-}=c_{+} / c_{-}, c_{-+}=c_{-} / c_{+}$).

$\left(c_{+}=1\right)$ and $\alpha^{2}$ equal to 0 or $100 \mathrm{~s}^{-2}$. We summarize the different configurations chosen in this document with the table 1 .

We use the HW-ABC coefficients of the table 2

\subsection{Single domain, dispersive and stratified material}

We show in this section different cases (figure 8) not to reconfirm the interest of the HW-ABC but to test our software with the HW-ABC: a case with a single domain with a dispersive material (the first value of the legend gives the $\mathrm{HW}-\mathrm{ABC}$ order for the propagative condition) and a case with a dispersive stratified domain with the HW-ABC with and without the evanescent condition (the second value of the legend gives the order of the evanescent condition). In the top domain, $c_{+}$is always equal to one. In the bottom domain, $c_{-}$can be equal to one or 0.3 . The dispersive coefficient $\alpha^{2}$ is always equal to 100 . The simulation time $T$ and the domain are larger according to the value of the inverse of $c_{-}$(first part of table 1). We use the orders 1, 2, 4, 8 and 16 with the HW-ABC coefficients given in the table 2 computed with the compatibility condition (6).

Remark: It is possible to inverse the ordering of the HW-ABC coefficients (for example for the order $4\left\{c_{+-}, c_{+-}, 1,1\right\}$ for the top domain and $\{1,1$, $\left.c_{-+}, c_{-+}\right\}$for the bottom domain with: $\left.c_{+-}=c_{+} / c_{-}, c_{-+}=c_{-} / c_{+}\right)$. So we get similar results. The intermediate orders are a little different keeping the same ranking but the last order is equivalent. We can add than these small differences disappear in the DDM computations. 

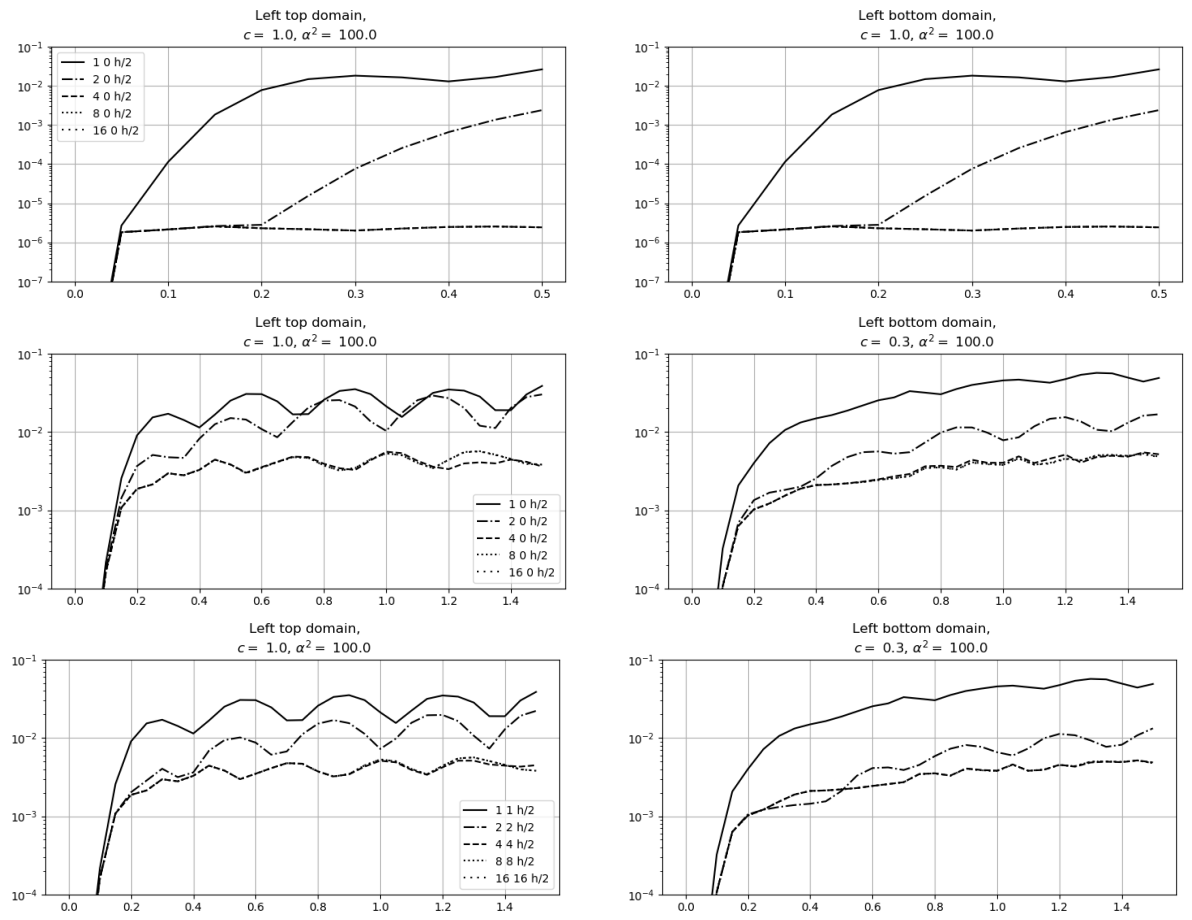

Figure 8: Single domain - dispersive $\left(\alpha^{2}=100\right)$ and stratified material: $L_{2}$ differences 40 with the space step equal to 0.005 . The abscissa is the time $(s)$. The first graph line is done with $\left\{c_{+}=c_{-}=1, T=0.5, l_{x}=l_{x 0}=0.6\right\}$, the second graph line is done with $\left\{c_{+}=1, c_{-}=0.3, T=3 \times 0.5 s, l_{x}=3 \times l_{x 0}\right\}$ and the last graph line is done with the previous values but with the $\mathrm{HW}-\mathrm{ABC}$ using the evanescent condition (with $\sigma=100$ ) (table 2 for the used HW-ABC coefficients).

In these cases, we compute the $L_{2}$ differences 40 for the top (with $c^{+}$) and bottom (with $c^{-}$) left domains $(x \leq 0)$. We get classical results. Only the case with the evanescent condition is different, we don't use here a high-order scheme as in [13]. The high orders of the HW-ABC are useful to get small errors between the solution $u$ and the reference solution. We can note better results with the evanescent condition for the intermediate orders, not for the bigger orders. But it is different for the SWR simulations (see next section).

The $\sigma$ coefficient (associated to the evanescent condition) has been chosen with the DDM process using the numerical simulations associated to the figure 9. We compute the $L_{2}$ differences for different $\sigma$ values for different SWR iterations for different HW-ABC orders with the evanescent condition (the first value of the legend gives the propagative order and the second the evanescent order). For the first iteration and the second iteration, the effect of the evanescent condition remains small but it appears. It is more interesting for the last iterations (4 and 8). We choose to use the $\sigma$ coefficient equal to 100 to get a compromise between the first (not yet the DDM process, just a more classical simulation with the $\mathrm{HW}-\mathrm{ABC}$ ) and the last SWR iterations. 

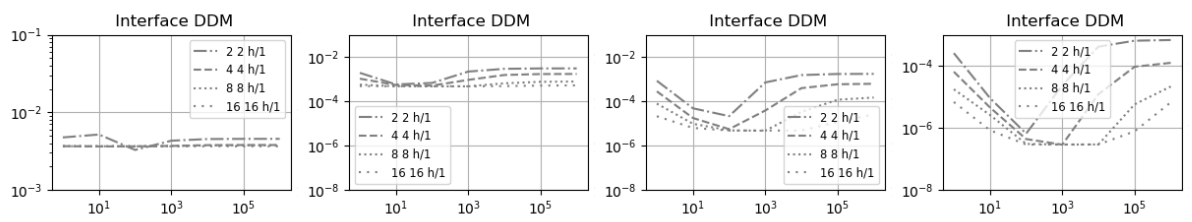

Figure 9: $L_{2}$ differences 40 on the interface between the two subdomains of the DDM process with the space step $h=0.01$ for the case $\left\{c_{+}=1, c_{-}=0.3, T=3 \times 0.5 s, l_{x}=3 \times l_{x 0}\right\}$ for different values $\left(10^{n}, n=0 . .6\right)$ of the $\sigma$ coefficient used in the evanescent condition for different SWR iterations (left to right : first (just the error of the left subdomain, classical use of the HW-ABC), 2, 4 and 8) and for different HW-ABC orders.

\subsection{Two domains (DDM), dispersive (or not) and stratified materials}

In this section, we show results of our domain decomposition applications. We start with some visualizations of a small part of the solutions to give a better understanding of our applications (next subsection). In the following subsections, we show the $L_{2}$ errors of our applications with and without time average. In the last subsection, we finish by a special bigger case.

\subsubsection{Visualizations of solutions}

In the figure 10, we illustrate the domain decomposition solutions at the eighth iteration of the SWR process with the none dispersive stratified case $\left\{c_{+}=1\right.$ for the top domain, $c_{-}=0.3$ for the bottom domain $\}$. Each graph line is for one time $(t \simeq 0.5,0.75,1 \mathrm{~s})$. The solution at $t$ equal to 1.5 second is shown in the middle line of the figure 11. The two first left graphs show a zoom of the amplitude SWR solution with filled iso-contours and the amplitude reference solution with dashed iso-contours, the first left graph with a colormap useful for the first moments of the simulation and the second left graph with a colormap more useful for the last moments. The right graph shows a zoom of the amplitude of the difference between the reference solution and the domain decomposition solution with a specific logarithmic scale colormap. The computation has been done with the more precise discretization in space and in time ( $h=0.005$ and $\delta t=0.0035)$ and with the order 32 for the HW-ABC without the evanescent condition. It is difficult to see the differences between the reference solution and the domain decomposition solution. Only in the right graph, we can see the small differences essentially on and near the interface between the two subdomains.

The figure 11 (composed in the same way as the previous figure) shows a comparison of the domain decomposition solutions computed with different $\mathrm{HW}$ ABC orders 4 and 32 without the evanescent condition and a HW-ABC with evanescent condition for the order 16 (and also for the propagative condition). As we see, the best solution is gotten for the case with the evanescent condition. We can see a difference in the middle graphs (with the reference solution for the top middle graph at $(x, y)=(0,-0.1))$ and of course with the right graphs giving the amplitude of the difference between the domain decomposition solution and the reference solution. For the order 4 without evanescent condition, the maximum value of the error amplitude is around $10^{-3}$. For the order 32 without evanescent condition, this value is around $10^{-5}$. For the best case with 

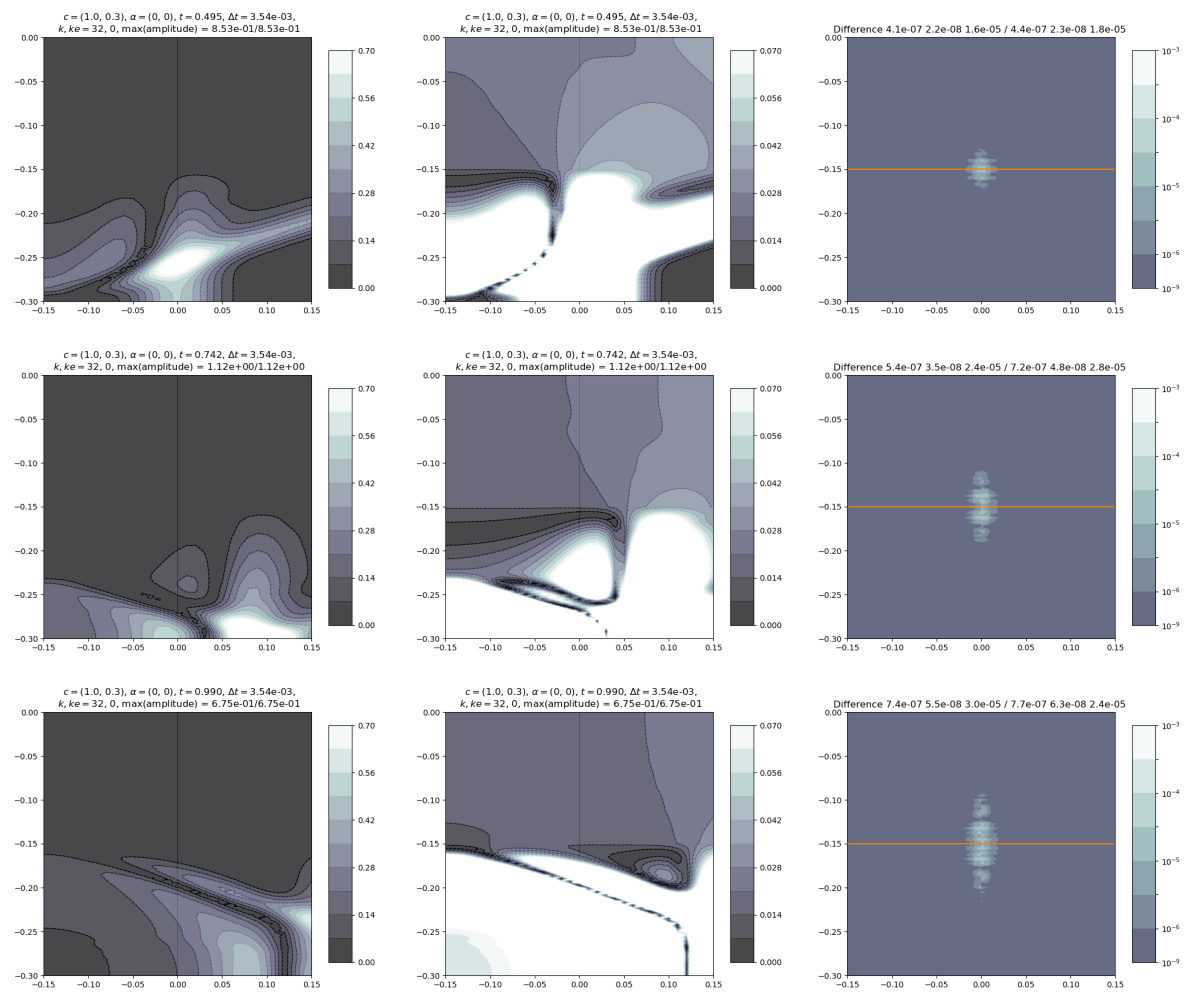

Figure 10: Illustrations of a domain decomposition none dispersive and stratified test for $\left\{c_{+}=1, c_{-}=0.3\right\}$ at the eighth iteration of the SWR process - Left and middle graphs: Zooms on amplitudes of the domain decomposition solution (filled iso-contours) and the reference solution (dashed iso-contours) for different times and two different colormaps - Right graphs: Associated zooms to show differences between the amplitudes of the domain decomposition solution and the reference solution with a specific log scale colormap.

the HW-ABC with evanescent condition, this value is around $10^{-7}$. So with our $\log$ scale colormap and in the best case, the differences disappear.

\subsection{2. $L_{2}$ errors with time average}

The figure 12 shows the $L_{2}$ errors 40 with time average following the SWR iterations for two none dispersive and stratified cases with $c_{+}$equal to one and $c_{-}$equal to 0.3 and 0.1 for HW-ABC with and without the evanescent condition. We use two space steps ( $h=0.01$ and $h / 2$ given in the legends) with the associated small time steps.

We get significant improvements between the first iteration and the last iteration. The ratio of the improvements is around $10^{6}$ for the best cases. As we see, the most important errors remain localized on the domain decomposition interface $(x=0)$. The other errors computed on the interface between materials and the domains are better. We must point out that the $L_{2}$ errors use also values of the right subdomain (with the values of the left subdomain). For the first iteration, we have a null solution for all the time in the right subdomain, the initial condition is fully positioned in the left subdomain. For the first iteration, 

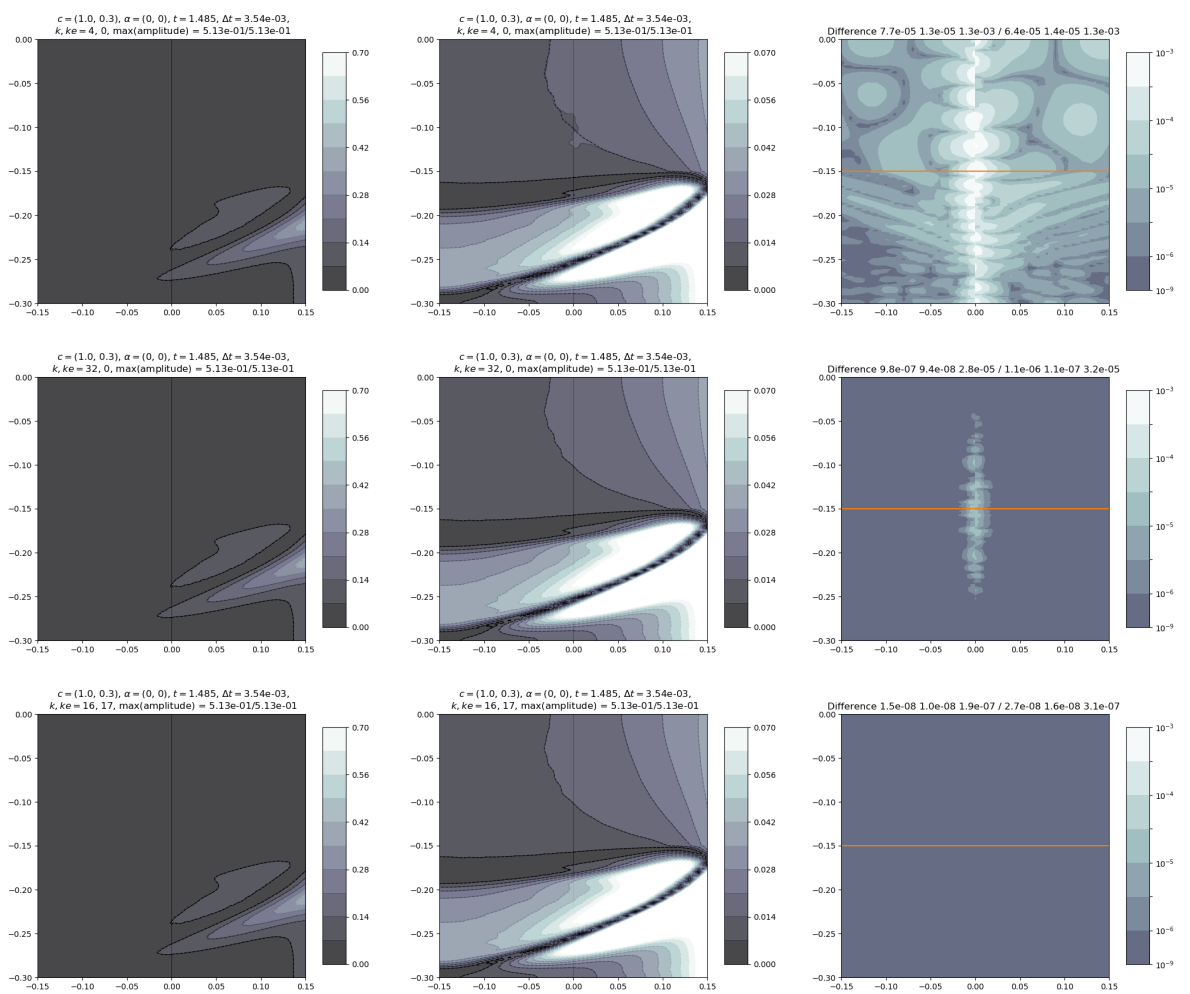

Figure 11: Comparison for one time $(t \simeq 1.5 \mathrm{~s})$ of two domain decomposition none dispersive and stratified tests for $\left\{c_{+}=1, c_{-}=0.3\right\}$ for the HW-ABC orders 4 (top line) and 32 (middle line) without evanescent condition and for the HW-ABC order 16 for the propagative and evanescent condition (bottom line) - The composition of the left, middle and right graphs is similar to the figure 10

the $L_{2}$ errors are aggravated by the null solution in the right subdomain

With five or six iterations, we get interesting results. Also we can note that a better space step is useful especially for the biggest orders 16 or 32 . The solution in the bottom domain (the slow domain) must be computed with more space precision (as we can see in the figure 10, the shapes of the solution are more complex than for the top domain) but also in the top domain for the local solutions near the interface between bi-materials [27].

The HW-SWR simulations with only the propagative condition gives interesting results. The contribution of the evanescent condition allows to get a more significant quality with a better cumulative order (propagative order (8) with evanescent order (8)) than the best results with just the propagative condition (order 32).

The figure 13 shows the similar results with the largest time steps, with and without the dispersive property $\left(\alpha^{2}=100\right)$. The organization of the figure is similar to that the figure 12. We give only the results with the best space step as we see in the legends $(h / 2$ with $h=0.01)$. For the dispersive case, the contribution of the evanescent condition is even more significant. 

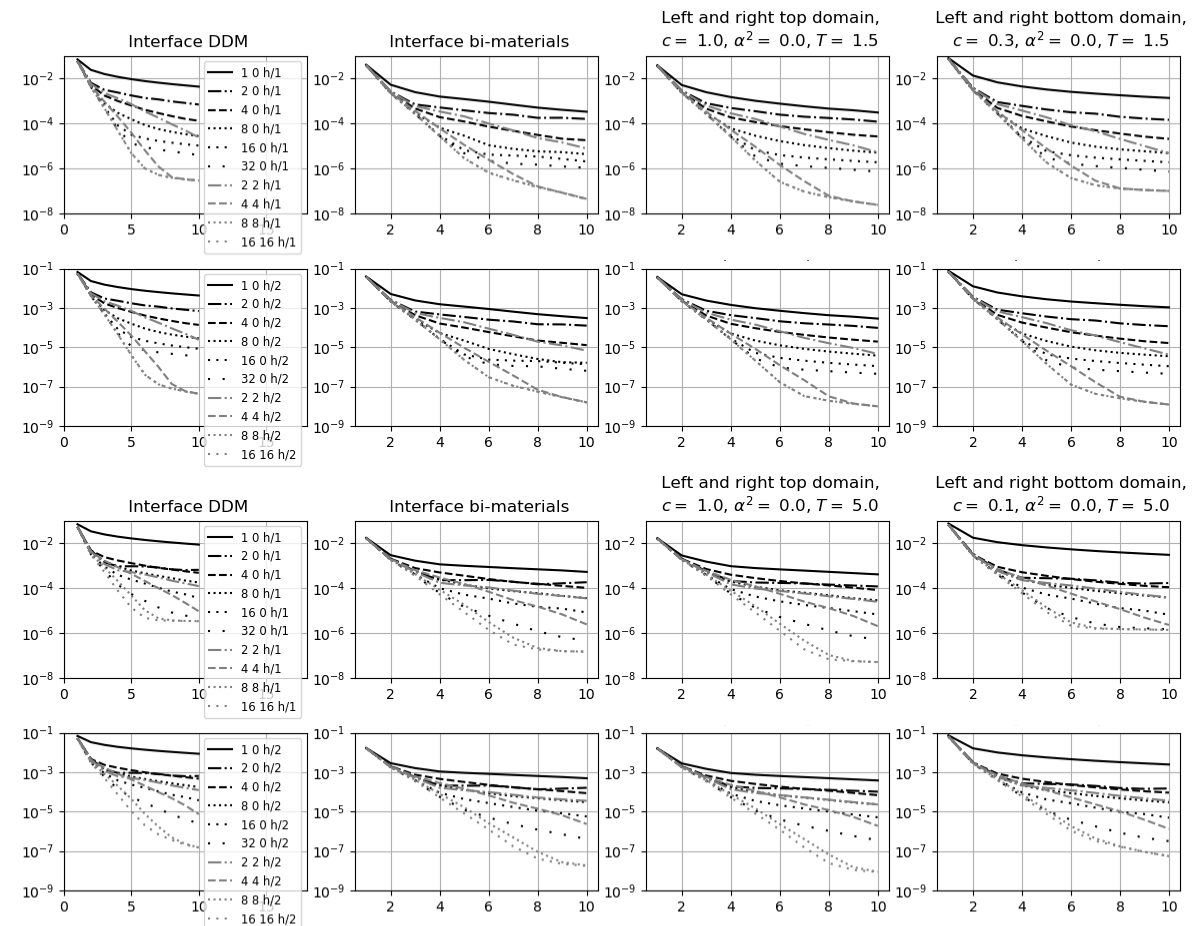

Figure 12: Small $\delta t$, non dispersive - $L_{2}$ average errors with mean in time / SWR iteration Two top graph lines $\left\{c_{+}=1, c_{-}=0.3, T=3 \times 0.5, l_{x}=3 \times l_{x 0}\right\}$ - Two bottom graph lines $\left\{c_{+}=1, c_{-}=0.1, T=10 \times 0.5, l_{x}=10 \times l_{x 0}\right\}$, from left to right for the domain decomposition interface $x=0$, the interface between the two different materials $y=-0.125$, the half left and half right top domain and the same for the bottom domain (legends - first number: HW-ABC order without evanescent condition - second number: order for the evanescent condition third "the label $h$ or $h / 2$ ": used space step, for all the graph line). 

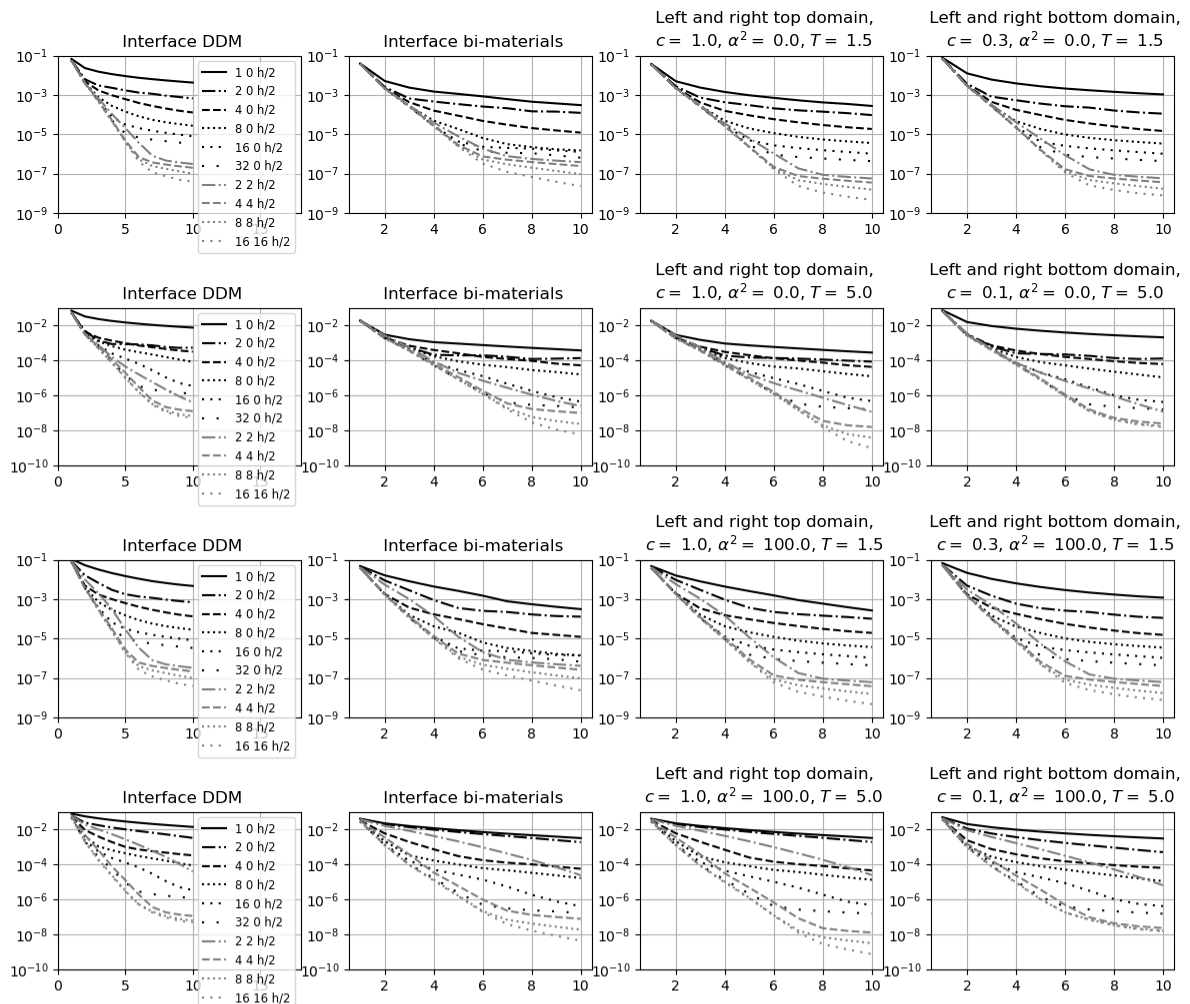

Figure 13: Big $\delta t$, non dispersive (first two graphs lines) and dispersive $\alpha^{2}=100$ (last two graphs lines) $-L_{2}$ time average errors / SWR iteration - Similar to the figure 12 just for the best space step.

\subsection{3. $L_{2}$ errors without time average}

The figure 14 allows to illustrate the behaviour of the $L_{2}$ errors 40 with the time for the cases $\left\{c_{+}=1, c_{-}=0.3, T=3 \times 0.5, l_{x}=3 \times l_{x 0}\right\}$ and $\left\{c_{+}=1, c_{-}=0.1, T=10 \times 0.5, l_{x}=10 \times l_{x 0}\right\}$ just for the HW-ABC with evanescent condition. We show the $L_{2}$ errors over time for the interfaces (left and right) between the two subdomains, for the bi-materials interfaces and the top and bottom domains for each subdomains (left and right). The two top graph lines are associated to the eighth SWR iteration for the case " $c_{-}=0.3$ ". The results can be 10 times better with a more flat behaviour between the low order (order 2 for the propagative and evanescent conditions) and the hight order (order 16 for the propagative and evanescent conditions) of the HW-ABC with propagative and evanescent conditions. The other two graph lines give the same results for the eighth (middle) and sixteenth (bottom) iterations for the case " $c_{-}=0.1 "$ for the orders 8 and 16 . This case seems a little unstable for the order 16 but the sixteenth iteration gives a better result to the same order.

240 But if we change the value of the coefficient $\sigma$ (with $\sigma=1000$ ), we get more stable results (figure 15). As we see in the figure 9, this value gives a better result for the biggest iteration and the biggest orders. We get similar results with the largest time steps (shown in the figure 16) and also with the dispersive cases. 

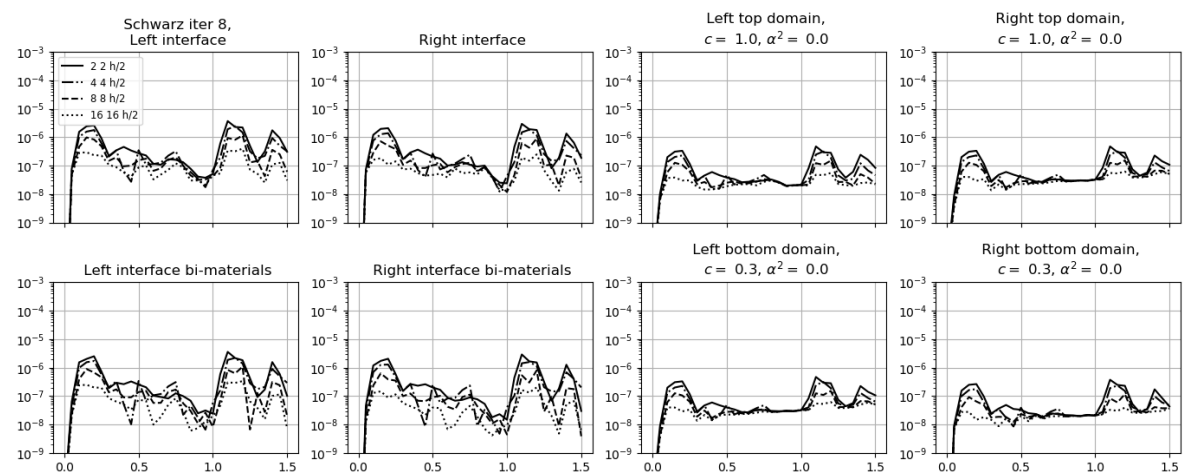

Right bottom domain

Schwarz iter 8,
Left interface

Right interface

Left top domain,
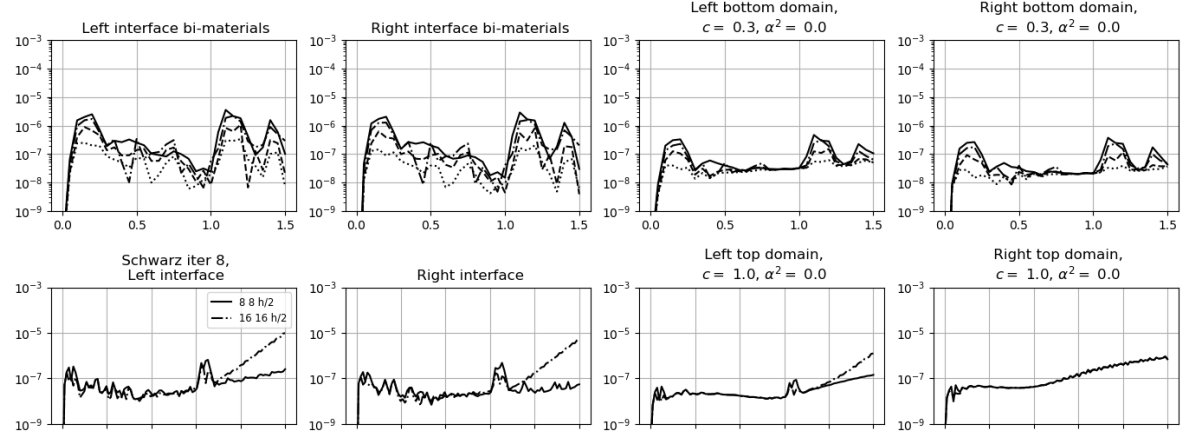

Right top domain,
$c=1.0, a^{2}=0.0$
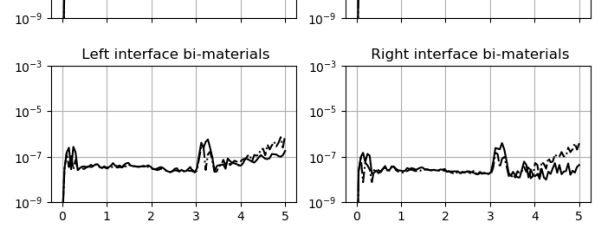

Left bottom domain.

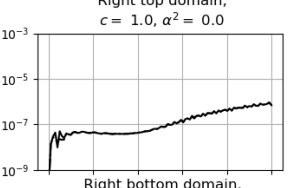

$c=0.1, \alpha^{2}=0.0$

Right bottom domain.
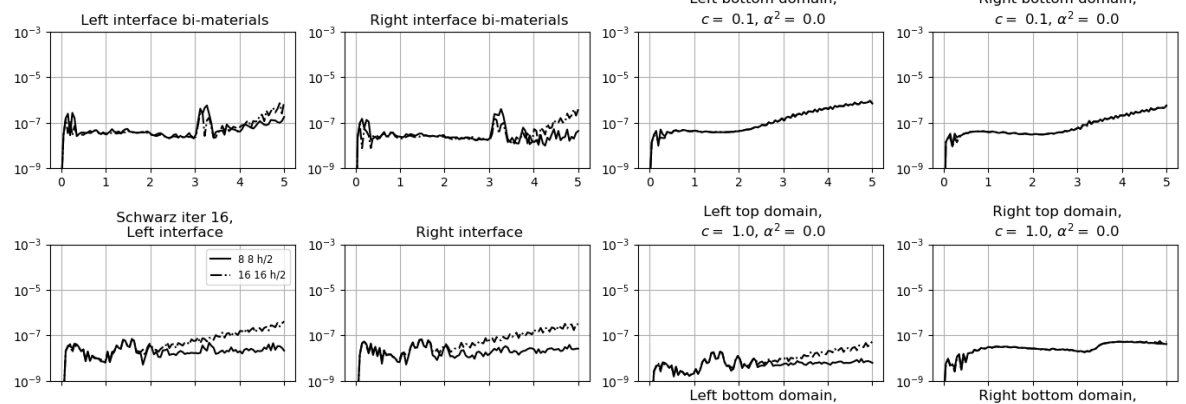

Right top domain.
$c=1.0, a^{2}=0.0$
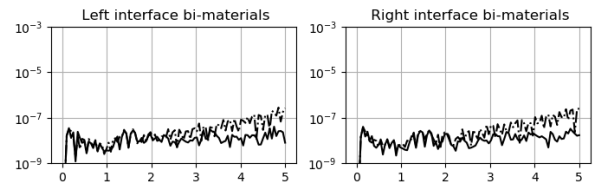

Left bottom domain,

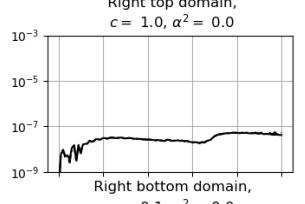

Right bottom domain,
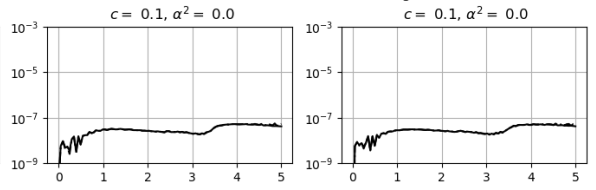

Figure 14: Small $\delta t$, non dispersive - $L_{2}$ average errors / time for the eighth SWR iterations associated to the first case $\left(c_{-}=0.3\right)$ of the figure 12 (two top graph lines) and for the eighth (two middle graph lines) and sixteenth (two bottom graph lines) SWR iterations associated to the second case $\left(c_{-}=0.1\right)$ of the figure 12

The first simulations of the DDM problems (with the initial coefficients $\lambda_{i}^{p}$ equal to zero (27) (29) ) give results with low precision also with the evanescent condition. For the left subdomain, these simulations are equivalent to the HWABC stratified (with layers) computations as in [13, but we don't use the same scheme. The authors of [13] use a high-order finite difference scheme (8th order) with a specific stabilization near the boundaries. With this method, the authors get best results. We use a classical implicit scheme possibly with big time steps. Our results are improved with the following iterations of the DDM process with the correction values of the coefficients $\lambda_{i}^{p}$. The following iterations of the DDM process allow to get better results with the evanescent condition as we see in the figure 11 . 

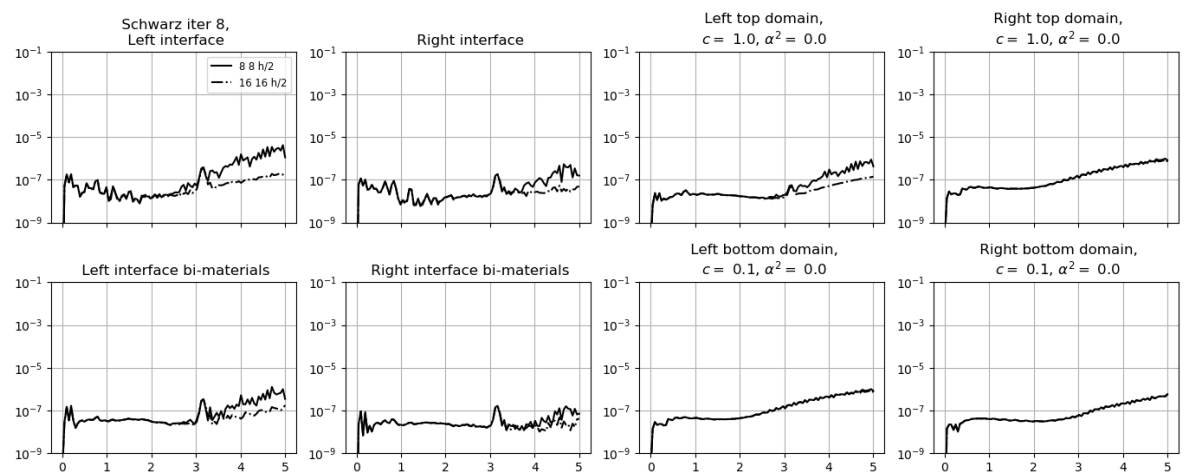

Left bottom domain

Right bottom domain
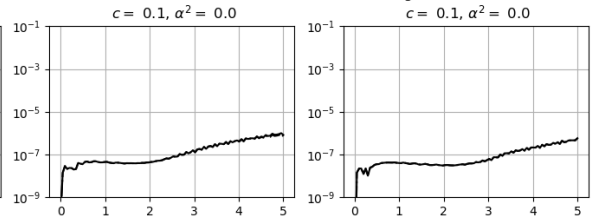

Schwarz iter 16
Left interface

Left top domain,

Right top domain
$c=1.0, a^{2}=0.0$
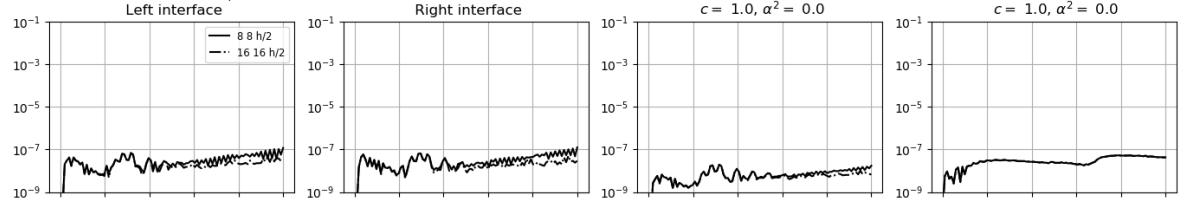

Left bottom domain,

Right bottom domain
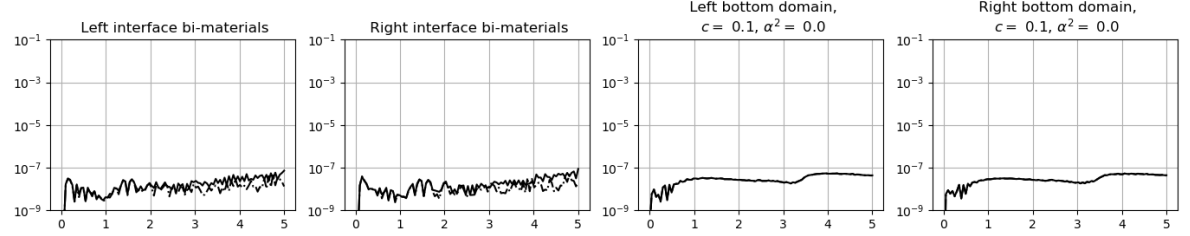

Figure 15: Small $\delta t$, non dispersive - $L_{2}$ average errors / time for the eighth (two middle graph lines) and sixteenth (two bottom graph lines) SWR iterations associated to the second case $\left(c_{-}=0.1\right)$ of the figure 12 with $\sigma=1000$.
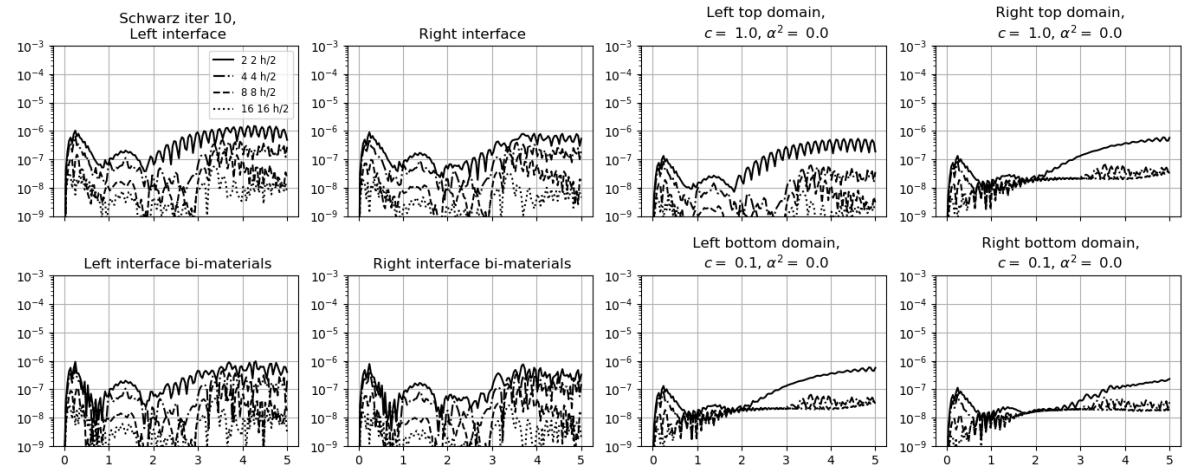

Right bottom domain,

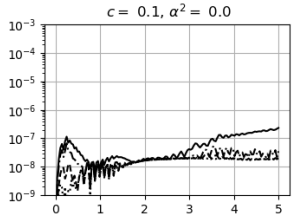

Figure 16: Big $\delta t$, non dispersive $-L_{2}$ average errors / time for the tenth SWR iteration associated to the second case $\left(c_{-}=0.1\right)$ of the figure 13

\subsubsection{Special bigger case}

To illustrate the possibilities of the HW-SWR process with the classical implicit scheme, we add here a new case with a larger gap between low and fast materials. We set always $c_{+}$equal to one for the fastest material. But we use $c_{-}$equal to 0.01 for the slowest material. The ratio $c_{+} / c_{-}$is equal to 100 . So with this important difference, we can get also an interesting result with a long 
time simulation (fifty seconds are useful for the initial waves to go out properly at the interface condition in the slow layer) as we see in the figure 17 . We use a specific time step equal to the previous big time step divided by ten to get a compromise between the two different materials. The best result has been done with the order 32 for the propagative and evanescent condition (with the used coefficient $\sigma$ equal to 1000). In this best case, the error remains small less than $10^{-7}$, more flat-equivalent for more long time at the eighth SWR iteration.

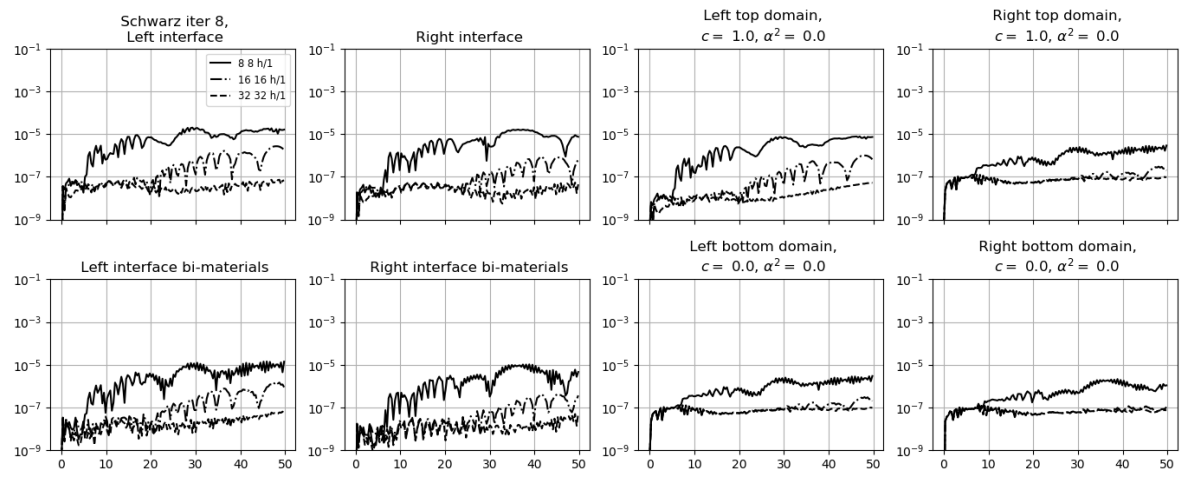

Figure 17: Big $\delta t$ divided by ten, non dispersive $-L_{2}$ average errors / time for the eighth SWR iteration associated to the case $\left(c_{-}=0.01 \mathrm{~m} . \mathrm{s}-1\right)$ with the space step $h$ equal to $0.01 \mathrm{~m}$ and the time step $\delta t$ equal to $0.07 \mathrm{~s}$ (with $T=50 \mathrm{~s}$ ) for three different HW-ABC orders (see the legend of the left-top graph).

\section{Conclusion}

In this paper, we have shown how to apply the high-order Absorbing Boundary Condition proposed by Hagstrom and Warburton to a stratified and dispersive wave equation solved with the Schwarz Waveform Relaxation algorithm to get DDM time-space solutions without overlapping with the classical implicit Newmark scheme. For that, we have few add-ons on each HW-ABC used as interface condition on the shared boundary of the two subdomains. We can use a cumulative process to get solutions.

With the implicit scheme, we have computed numerical simulations using a small time step designed to the fast material and a big time step designed to the slow material. We get similar numerical results between these two time steps. Classically to get computations with the Hagstrom-Warburton Absorbing Boundary Condition, the authors prefer use small time steps. We apply it here also with a larger time step for DDM processes.

A sufficient space discretization remains nevertheless useful. For example with the stratified cases, we must use a sufficiently small space discretization to get interesting precisions, in particular to get similar precisions between fast and slow domains.

Our last test, with a larger gap between low and fast materials, allows to imagine that this method can be applied to a wider range of problems. 


\section{References}

[1] B. Engquist, A. Majda, Absorbing Boundary Conditions for the Numerical Simulation of Waves, Math. of comp., Vol. 31 No 139, pp. 629-651, 1977.

[2] A. Bayliss, E. Turkel, Radiation Boundary Conditions for Wave-Like Equations, Pure and Applied Math., Vol. 33, 707-725, 1980.

[3] R. L. Higdon, Absorbing Boundary Conditions for Difference Approximations to the Multi-Dimensional Wave Equation, Math. of comp., Vol.47 No 176, pp. 437-459, 1986.

[4] R. L. Higdon, Numerical Absorbing Boundary Conditions for the Wave Equation, Math. of comp., Vol. 49 No 179, pp. 65-90, 1987.

[5] F. Collino, High order absorbing boundary conditions for wave propagation models: straight line boundary and corner cases, in: R. Kleinman et al. (Eds.), Proc. 2nd Int. Conf. on Mathematical and Numerical Aspects of Wave Propagation, SIAM, Delaware, pp. 161-171, 1993.

[6] J.P. Bérenger, A perfectly matched layer for the absorption of electromagnetic waves, J. Comput. Phys. 114, pp. 185-200, 1994.

[7] M.J. Grote, J.B. Keller, Exact nonreflecting boundary conditions for the time dependent wave equation, SIAM J. Appl. Math. 55, pp.280-297, 1995.

[8] T. Hagstrom, S.I. Hariharan, A formulation of asymptotic and exact boundary conditions using local operators, Appl. Numer. Math. 27, pp. 403-416, 1998.

[9] M.N. Guddati, J.L. Tassoulas, Continued-fraction absorbing boundary conditions for the wave equation, J. Comput. Acoust. 8, pp. 139-156, 2000.

[10] D. Givoli, B. Neta, High-order non-reflecting boundary scheme for timedependent waves, J. of Comp. Phys. 186, pp. 24-46, 2003.

[11] Th. Hagstrom, T. Warburton, A new auxiliary variable formulation of high-order local radiation boundary conditions: corner compatibility conditions and extensions to first-order systems, Wave Motion, Vol 39 No 4, pp. 327-338, 2004.

[12] D. Givoli, High-order local non-reflecting boundary conditions: a review, Wave Motion 39, pp. 319-326, 2004.

[13] Th. Hagstrom, A. Mar-Or, D. Givoli, High-order local absorbing conditions for the wave equation: Extensions and improvements, Comp. Phys. 227, pp. 3322$3357,2008$.

[14] E. Bécache, D. Givoli, Th. Hagstrom, High-order Absorbing Boundary Conditions for anisotropic and convective wave equations, Comp. Phys. 229, pp. 1099-1129, 2010 .

[15] H. A. Schwarz, Über einen Grenzübergang durch alternierendes Verfahren , Vierteljahrsschrift der Naturforschenden Gesellschaft in Zürich, 15, pp. 272-286, 1870 .

[16] P.L. Lions - On the Schwarz alternating method 1. SIAM, 1st int. symp. on DDM for partial diff. eq., 1988.

[17] J. S. Przemieniecki, Matrix structural analysis of substructures, Am. Inst. Aero. Astro. J.1, pp. 138-147, 1963. 
[18] Achi Brandt, Multi-Level Adaptive Solutions to Boundary-Value Problems, Mathematics of Computation, Vol. 31, No. 138, pp. 333-390, 1977.

[19] E. Lelarasmee, A. E. Ruehli, A. L. Sangiovanni-Vincentelli, The waveform relaxation method for time-domain analysis of large scale integrated circuits, IEEE Trans. on CAD of IC and Syst., 1, pp. 131-145, 1982.

[20] C. Farhat and F. X. Roux, A method of finite element tearing and interconnecting and its parallel solution algorithm, Internat. J. Numer. Meths. Engrg. 32, 12051227 (1991).

[21] M. Bjørhus, On domain decomposition, subdomain iteration and waveform relaxation, Ph.D. thesis, University of Trondheim, Norway, 1995.

[22] M. J. Gander and A. M. Stuart, Space time continuous analysis of waveform relaxation for the heat equation, SIAM J. 19, pp. 2014-2031, 1998.

[23] M. Gander, L. Halpern, Méthodes de décomposition de domaines pour l'équation des ondes en dimension 1, C.R. Acad. Sci. Paris, t. 332, Série 1, pp. 1-4, 2001.

[24] M. J. Gander, L. Halpern, F. Nataf, Optimal Schwarz Waveform Relaxation for the one dimensional wave equation, SIAM Num. Anal., Vol 41 No 5, pp. 1643 $1681,2003$.

[25] M J. Gander, L. Halpern, Absorbing Boundary Conditions for the Wave Equation and Parallel Computing, Math. of comp., Vol. 74, No 249, pp. 153-176, 2004.

[26] J. Fabre, Ondes, http://thual.perso.enseeiht.fr/otond/fabre/poly/Ondes.pdf.

[27] Ph. Destuynder, O. Wilk, Local Waves in heterogenous media and their control - Proceedings of the 4th European Workshop on Structural Health Monitoring, Cracow, Poland, July 2-4 2008 - Ed.: T. Uhl, W. Ostachowicz, J. Holnicki-Szulc - ISBN 978-1-932078-94-7 - DEStech publications - pp. 682-691, 2008. 